\title{
Article \\ Comparison of Surface Properties of Sepiolite and Palygorskite: Surface Energy and Nanoroughness
}

\author{
Ricardo Almeida ${ }^{1}$, Eduardo Ferraz ${ }^{2,3} \mathbb{D}^{\text {, Julio Santarén }}{ }^{4}\left(\mathbb{D}\right.$ and José A. F. Gamelas ${ }^{1, * \mathbb{C}}$ \\ 1 CIEPQPF-Chemical Process Engineering and Forest Products Research Centre, \\ Department of Chemical Engineering, University of Coimbra, Rua Sílvio Lima, Pólo II, \\ PT-3030-790 Coimbra, Portugal; ricardsalmeida00@gmail.com \\ 2 Techn\&Art, Polytechnic Institute of Tomar, Quinta do Contador, Estrada da Serra, \\ PT-2300-313 Tomar, Portugal; ejmoferraz@ipt.pt \\ 3 Geobiotec, Geosciences Department, Campus Universitário de Santiago, University of \\ Aveiro, PT-3810-193 Aveiro, Portugal \\ 4 Tolsa, SA, Research \& Technology for New Businesses, Ctra. de Madrid a Rivas Jarama, 35, \\ ES-28031 Madrid, Spain; jsantaren@tolsa.com \\ * Correspondence: jafgas@eq.uc.pt
}

Citation: Almeida, R.; Ferraz, E.; Santarén, J.; Gamelas, J.A.F Comparison of Surface Properties of Sepiolite and Palygorskite: Surface Energy and Nanoroughness. Nanomaterials 2021, 11, 1579. https:// doi.org/10.3390/nano11061579

Academic Editor: Alexey Pestryakov

Received: 7 April 2021

Accepted: 10 June 2021

Published: 16 June 2021

Publisher's Note: MDPI stays neutral with regard to jurisdictional claims in published maps and institutional affiliations.

Copyright: (c) 2021 by the authors. Licensee MDPI, Basel, Switzerland. This article is an open access article distributed under the terms and conditions of the Creative Commons Attribution (CC BY) license (https:// creativecommons.org/licenses/by/ $4.0 /)$.

\begin{abstract}
The surface properties of two sepiolite samples and one palygorskite sample were compared using inverse gas chromatography (IGC). Samples were previously conditioned at appropriate temperatures for the removal of all zeolitic water. Dispersive (or Lifshitz-van der Waals) component of the surface energy $\left(\gamma_{\mathrm{s}}{ }^{\mathrm{d}}\right)$, specific interactions $\left(-\Delta \mathrm{G}_{\mathrm{a}}{ }^{\mathrm{s}}\right)$ with $\pi$ electron donor bases (1-alkenes), and nanomorphology indices $\left(\mathrm{IM} \chi_{\mathrm{T}}\right)$ based on the injections of cycloalkanes and a branched alkane were measured. From IGC data, at $240{ }^{\circ} \mathrm{C}$, it was found that the palygorskite was clearly distinguished from the sepiolites. The palygorskite possessed a lower $\gamma_{s}{ }^{d}$, larger $-\Delta G_{a}{ }^{s}$ with 1-alkenes, and remarkably higher $\mathrm{IM} \chi_{\mathrm{T}}$. Slight differences could also be observed between the two sepiolite samples with the same origin. The results were rationalized in terms of the structural features of the two studied minerals. The larger channels of the sepiolite allow for a better insertion of the $n$-alkanes (longer retention times) while excluding the bulkier probes, such as cyclooctane or 2,2,4-trimethylpentane. Accordingly, the corresponding $\gamma_{\mathrm{s}}{ }^{\mathrm{d}}$ values were larger and the IM $\chi_{\mathrm{T}}$ values were lower (higher surface nanoroughness) for the sepiolites. Regarding Lewis acid-base properties, all the sample's surfaces evidenced a very strong amphoteric character. The present results highlight the potential of the evaluated samples for, e.g., adsorption processes with volatile organic compounds or matrix-filler interactions regarding the production of composite structures with Lewis acid-base matrices.
\end{abstract}

Keywords: adsorption; aluminosilicate; attapulgite; clay minerals; fibrous minerals; inverse gas chromatography

\section{Introduction}

Sepiolite and palygorskite are fibrous clay minerals composed of discontinuous structural blocks, alternating with structural tunnels. Each block has a 2:1 phyllosilicate structure (two silica tetrahedral sheets with a central octahedral sheet) and is linked to the next inverted tetrahedral sheet by $\mathrm{Si}-\mathrm{O}-\mathrm{Si}$ bonds [1,2]. Sepiolite is a trioctahedral mineral with the ideal formula $\mathrm{Si}_{12} \mathrm{O}_{30} \mathrm{Mg}_{8}(\mathrm{OH})_{4}\left(\mathrm{OH}_{2}\right)_{4} \cdot 8 \mathrm{H}_{2} \mathrm{O}$, having all the octahedral sites occupied by magnesium. In contrast, palygorskite, with the ideal formula $\mathrm{Si}_{8} \mathrm{O}_{20}\left(\mathrm{Al}_{2} \mathrm{Mg}_{2}\right)(\mathrm{OH})_{2}\left(\mathrm{OH}_{2}\right)_{4} \cdot 4 \mathrm{H}_{2} \mathrm{O}$, has a dioctahedral character, and only four of the five octahedral sites are occupied, mainly with magnesium and aluminum ions [2,3]. The structural tunnels of these two minerals have cross-section dimensions of $1.06 \times 0.37 \mathrm{~nm}^{2}$ and $0.64 \times 0.37 \mathrm{~nm}^{2}$ for sepiolite and palygorskite, respectively, and inside them, there are exchangeable cations and zeolitic water. When the zeolitic water of these tunnels is removed by heating, the sorption capacity is increased and small organic molecules 
could also be adsorbed [2]. Due to the special sorptive, colloidal-rheological, and catalytic properties of these two minerals, they have attracted a growing interest for a wide range of applications $[4,5]$.

Inverse gas chromatography (IGC) is a powerful technique for the characterization of a material's surface properties. This tool can be used to study a wide variety of materials including polymers, cellulosic materials, minerals and inorganic materials, pharmaceuticals and medical products, supported catalysts, and microporous materials [6-8]. IGC is a variation of conventional gas chromatography (GC), and the main distinguishing factor between both techniques is related to the mobile and stationary phases. In IGC, the solid material under investigation (stationary phase) is packed into the column and a volatile probe molecule (single gas or vapor) of known characteristics is injected into the flow of an inert carrier gas and passes through the column $[7,8]$. From the determined retention times of different vapor probes on the surface of the solid material, a wide range of physicochemical properties can be obtained, including dispersive component of the surface energy of the material, specific components of the free energy, enthalpy and entropy of adsorption of the probes, nanoroughness, Flory-Huggins parameters, and glass transition temperatures. IGC is a very accurate and versatile characterization tool. In particular, IGC can overcome the contact angle measurement limitations in the analysis of porous, rough, and heterogeneous surfaces $[6,7,9]$.

Some studies have already been carried out to investigate the surface properties of sepiolite and palygorskite by IGC.

Morales et al. (1991) used IGC analysis to determine the enthalpy and entropy of adsorption of $n$-alkanes $(n=6,8$, and 9) and of two polar probes (dichloromethane and furane) and the dispersive component of the surface energy $\left(\gamma_{\mathrm{s}}{ }^{\mathrm{d}}\right)$ and to assess briefly the Lewis acid-base characteristics of a sepiolite sample pretreated with hydrogen peroxide. It was observed in the range of evaluated temperatures $\left(100-130^{\circ} \mathrm{C}\right)$ that the $\gamma_{\mathrm{s}}{ }^{\mathrm{d}}$ of sepiolite increased with increasing temperature, varying from 54 to $74 \mathrm{~mJ} \mathrm{~m}^{-2}$. Based on the measured acidity and basicity constants $\left(\mathrm{K}_{\mathrm{A}}\right.$ and $\mathrm{K}_{\mathrm{D}}$, respectively), the authors proposed that the sample surface was slightly more acidic than basic [10]. A few years later, Așkin and Yazici (2005) estimated several surface properties of another sepiolite sample in a quite different range of temperatures $\left(320-350^{\circ} \mathrm{C}\right)$ by measuring the retention times of different nonpolar and polar probes. The results showed that $\gamma_{\mathrm{s}}{ }^{\mathrm{d}}$ values decreased with increasing temperature $\left(320-350{ }^{\circ} \mathrm{C}\right)$, ranging from $84\left(320^{\circ} \mathrm{C}\right)$ to $71 \mathrm{~mJ} \mathrm{~m}{ }^{-2}\left(350{ }^{\circ} \mathrm{C}\right)$. These authors also made a comparison with other clay materials concerning the dispersive component of the surface energy and concluded that $\gamma_{\mathrm{s}}{ }^{\mathrm{d}}$ values for sepiolite were rather lower than those obtained for kaolinite, illite, or bentonite. Their studies also pointed to a prevalent acidic character of the sepiolite surface, under the measured conditions [11]. Lazarevic et al. (2009) [12] studied the adsorption properties of sepiolite by IGC at zero and finite surface coverage between 210 and $240{ }^{\circ} \mathrm{C}$. From IGC data at zero coverage, they found that dispersive component values decreased with temperature increase, ranging from $150\left(210^{\circ} \mathrm{C}\right)$ to $130 \mathrm{~mJ} \mathrm{~m}{ }^{-2}\left(240^{\circ} \mathrm{C}\right)$. However, based on the calculation of the $\mathrm{K}_{\mathrm{A}}$ and $\mathrm{K}_{\mathrm{D}}$ constants, they proposed that sepiolite surface had a prevalent basic character, in contrast to what was concluded in the other previous works $[10,11]$.

Boudriche et al. (2010) evaluated the influence of a grinding process on the surface properties of palygorskite by IGC. The dispersive component at $130{ }^{\circ} \mathrm{C}$, before grinding, was found to be $164 \mathrm{~mJ} \mathrm{~m}^{-2}$. With the grinding, the $\gamma_{\mathrm{s}}^{\mathrm{d}}$ values decreased slightly (down to $\left.143 \mathrm{~mJ} \mathrm{~m}^{-2}\right)$. From the calculation of the nanomorphology index $\left(\mathrm{IM} \chi_{\mathrm{T}}\right)$ of 2,3,4trimethylpentane and cyclooctane, the authors demonstrated that the surface morphology of palygorskite was not significantly influenced by the grinding process [13].

The aim of the present work was to make a comparative study of two sepiolite samples and one palygorskite sample (provided by TOLSA), measured in the same temperature conditions and after an appropriate preconditioning. These samples were thoroughly analyzed by IGC, which included the determination of the dispersive (Lifshitz-van der Waals) component of the surface energy $\left(\gamma_{\mathrm{s}}{ }^{\mathrm{d}}\right)$, specific component of the probes' free energy 
of adsorption $\left(-\Delta \mathrm{G}_{\mathrm{a}}^{\mathrm{s}}\right)$, and surface nanomorphology indices $\left(\mathrm{IM} \chi_{\mathrm{T}}\right)$, by injecting a wide range of probes ( $n$-alkanes, 1 -alkenes, cycloalkanes, and a branched alkane). The obtained results could be of interest for optimizing adsorption processes using this clay mineral type or valuable in the area of the production of composites, where the determined parameters can be used to predict compatibility between the clay surface and other components.

\section{Materials and Methods}

\subsection{Clays}

The geogenic sepiolite, supplied by Tolsa, SA (Madrid, Spain), was taken from the deposit of Vallecas-Vicálvaro (Madrid, Spain). The raw material was submitted to dry micronization using a jet mill to break the fiber bundles down into micron-size particles (sepiolite 1 (Sep. 1)) or to wet micronization producing an extensive deagglomeration of the bundles without affecting their aspect ratio (sepiolite 2 (Sep. 2)).

The geogenic palygorskite (Pal) was extracted from a deposit located in the department of M'bour, region of Thiès, south of Dakar (Senegal), and was also supplied by Tolsa, SA (Madrid, Spain). The raw material was preprocessed by micronization in a roller mill.

The general characterization of these fibrous clay samples was reported in detail elsewhere $[14,15]$.

Powder X-ray diffractograms were obtained using a Philips X'Pert MPD diffractometer (Amsterdam, the Netherlands), with $\mathrm{CoK} \alpha$ radiation $(\lambda=1.7903 \AA)$. Diffractograms were collected by the counting method (step $0.025^{\circ}$ and time $1.0 \mathrm{~s}$ ) in the $2 \theta$ range of $5-60^{\circ}$. Crystalline phases were discriminated by comparison with reference diffractograms from the International Centre for Diffraction Data.

Thermogravimetric plots were acquired using a TA Instruments simultaneous TGADSC thermal analyzer, model SDT Q600 (TA Instruments, New Castle, DE, USA). The samples were heated from room temperature up to $100{ }^{\circ} \mathrm{C}$, at a rate of $10^{\circ} \mathrm{C} / \mathrm{min}$, under a nitrogen atmosphere.

Brunauer, Emmett, and Teller (BET) specific surface area was determined by nitrogen adsorption at $77 \mathrm{~K}$ using a Micromeritics Gemini V analyzer (Micromeritics Instruments, Norcross, GA, USA). The samples were previously degassed at $124{ }^{\circ} \mathrm{C}$ under nitrogen flow for $12 \mathrm{~h}$.

\subsection{Inverse Gas Chromatography (IGC)}

Prior to IGC analysis, the powders of the two sepiolite samples and the palygorskite sample were compacted using a hydraulic press. The obtained pressed disc was then crumbled and sieved between 850 and $500 \mu \mathrm{m}$ before being packed in the IGC column. This procedure allowed a sufficient gas flow rate in the IGC column to be obtained.

The IGC analysis was performed using a DANI GC 1000 digital pressure control gas chromatograph equipped with a hydrogen flame ionization detector. Stainless steel columns, $0.4 \mathrm{~m}$ long and $0.4 \mathrm{~cm}$ inside diameter, were washed with acetone, dried, and passed through compressed air before packing. For these analyses, three different columns were run for each sample: $2.3-2.7 \mathrm{~g}$ of sepiolite 1, 2.0-2.4 $\mathrm{g}$ of sepiolite 2, and 2.7-3.2 $\mathrm{g}$ of palygorskite were packed inside the gas chromatograph column. The packed columns were shaped to fit the detector/injector geometry of the instrument and, after that, were conditioned overnight $(14 \mathrm{~h})$ at $290^{\circ} \mathrm{C}$ for the sepiolite samples and at $240{ }^{\circ} \mathrm{C}$ for the palygorskite sample, under a helium flow ( $p=0.05 \mathrm{bar}$ ), before any measurements were made. Measurements were then carried out at $240{ }^{\circ} \mathrm{C}$, for all samples, with injector and detector kept at 180 and $200{ }^{\circ} \mathrm{C}$, respectively. Helium (high purity, 99.99\%) was used as the carrier gas with flow rates (measured with a digital flow meter) between 55 and $92 \mathrm{~mL} \mathrm{~min}^{-1}$. The helium flow rate was controlled in all series of experiments. Small quantities of probe vapor $(<1 \mu \mathrm{L})$ were injected into the carrier gas, allowing work under infinite dilution conditions. $n$-alkanes (n-pentane $\left(\mathrm{C}_{5}\right), n$-hexane $\left(\mathrm{C}_{6}\right), n$-heptane $\left(\mathrm{C}_{7}\right)$, and $n$-octane $\left.\left(\mathrm{C}_{8}\right)\right)$, 1-alkenes (1-pentene and 1-hexene), cycloalkanes (cyclohexane (cyclo 6) and cyclooctane (cyclo 8)), and a branched alkane (2,2,4-trimethylpentane (2,2,4-TMP)) were the probes 
used for the IGC data collection. Tetrahydrofuran, diethyl ether, ethyl acetate, and acetone were also injected, but due to their strong interaction with the studied materials, they could not be eluted through the column. On the other hand, injections of trichloromethane and dichloromethane provided irreproducible signals (null or very weak), which were not further considered for the analysis. All probes used were of chromatographic grade. Methane was used as the reference probe. The retention times $\left(t_{r}\right)$ were the average of three injections and were determined by the Conder and Young method [16]. Coefficient of variation between injections was lower than $5 \%$. From the retention time data, the dispersive component of the surface energy, the specific component of the probes' free energy of adsorption, and the surface nanomorphology indices were determined.

\subsection{IGC Theory}

The principles and the mathematical aspects of the IGC theory in infinite dilution conditions have been widely described in the literature [6-8,17]. Briefly, by the Schultz and Lavielle approach, the dispersive component of the surface energy (Lifshitz-van der Waals component, in the strict sense) for the solid material under analysis may be estimated from the slope of the linear fit of $R T \ln \left(\mathrm{V}_{\mathrm{n}}\right)$ as a function of $2 \mathrm{~N} \cdot a\left(\gamma_{1} \mathrm{~d}\right)^{0.5}$ obtained with the injection of apolar probes, usually $n$-alkanes (Equation (1)) [18].

$$
\mathrm{R} \operatorname{Tln}\left(\mathrm{V}_{\mathrm{n}}\right)=\sqrt{\gamma_{\mathrm{s}}^{\mathrm{d}}} 2 \mathrm{~N} \cdot a \sqrt{\gamma_{1}^{\mathrm{d}}}+\mathrm{K}
$$

where $\mathrm{R}$ is the ideal gas constant; $\mathrm{T}$ is the column absolute temperature; $\mathrm{V}_{\mathrm{n}}$ is the net retention volume of the probe; $\mathrm{N}$ is the Avogadro number; $a$ is the molecular surface area of the probe, $\gamma_{\mathrm{s}}{ }^{\mathrm{d}}$ and $\gamma_{1}{ }^{\mathrm{d}}$ are the dispersive components of the surface free energy of the interacting solid material and probe, respectively; and $\mathrm{K}$ is a constant that is dependent on the chosen reference state.

The dispersive component of the surface energy can also be estimated by the Dorris and Gray approach. Here, the $\gamma_{\mathrm{s}}{ }^{\mathrm{d}}$ value is determined from the difference in the free energy of adsorption due to the introduction of an additional methylene group into the carbon chain of an $n$-alkane probe (Equation (2)) [19].

$$
\gamma_{\mathrm{s}}^{\mathrm{d}}=\frac{\left[\operatorname{RT} \ln \frac{\mathrm{V}_{\mathrm{n}}^{\left(\mathrm{C}_{\mathrm{n}+1} \mathrm{H}_{2 \mathrm{n}+4}\right)}}{\mathrm{V}_{\mathrm{n}}^{\left(\mathrm{C}_{\mathrm{n}} \mathrm{H}_{2 \mathrm{n}+2}\right)}}\right]^{2}}{4 \mathrm{~N}^{2}\left(a_{\mathrm{CH}_{2}}\right)^{2} \gamma_{\mathrm{CH}_{2}}}
$$

where $a_{\mathrm{CH}_{2}}$ is the molecular area of the methylene group $\left(0.06 \mathrm{~nm}^{2}\right)$ and $\gamma_{\mathrm{CH}_{2}}$ is the surface energy of a solid entirely composed of methylene groups (Equation (3)).

$$
\gamma_{\mathrm{CH}_{2}}=35.6+0.058(293.15-\mathrm{T})
$$

For probes able to establish molecular interactions with the solid material other than dispersive ones (e.g., Lewis acid-base interactions), there is a corresponding specific component contribution, $\Delta \mathrm{G}_{\mathrm{a}}$ s, to the overall free energy of adsorption, $\Delta \mathrm{G}_{\mathrm{a}}$ [17]. The $\Delta \mathrm{G}_{\mathrm{a}}{ }^{\mathrm{s}}$ contribution can be estimated by calculating the difference between the experimental value of $R T \ln \left(V_{n}\right)$ obtained for the respective probe (Lewis acidic, basic, or amphoteric) and the corresponding $R T \ln \left(V_{n}\right)$ for the reference $n$-alkane probe (Equation (4)).

$$
\Delta \mathrm{G}_{\mathrm{a}}^{\mathrm{s}}=-\mathrm{R} \ln \frac{\mathrm{V}_{\mathrm{n}}}{\mathrm{V}_{\mathrm{n}, \mathrm{ref}}}
$$

To evaluate the nanoroughness of the studied clayey materials, the nanomorphology index $\left(\mathrm{IM} \chi_{\mathrm{T}}\right)$ was calculated according to two different approaches. The Brendlé and Papirer approach compares the measured $\chi_{\exp }$ with the $\chi_{\mathrm{T}}$ topological index (Equation (5)) [20]. Here, $\chi_{\mathrm{T}}$ is the previously defined topological index of a branched alkane or cycloalkane, determined using Wiener's indices, and represents the number of carbon atoms of a hypo- 
thetical linear alkane that would interact with the surface of an ideal solid (without surface roughness) the same way as the branched alkane or the cycloalkane. $\chi_{\text {exp }}$ is the experimental value calculated for this topological index based on the measurement of the retention times of the nonlinear alkane and the $n$-alkanes on the solid material under analysis.

$$
\operatorname{IM} \chi_{\mathrm{T}}=100 \times \frac{\left(\chi_{\exp }-\chi_{\mathrm{T}}\right)}{\chi_{\mathrm{T}}}
$$

Typically, for less rough surfaces, $\chi_{\exp }$ approaches $\chi_{\mathrm{T}}$ and $\mathrm{IM} \chi_{\mathrm{T}}$ tends to $0 \%$; on the contrary, the rougher the surface is, the more negative the IM $\chi_{\mathrm{T}}$ value is.

Balard et al. (2000) suggested another approach for $\mathrm{IM} \chi_{\mathrm{T}}$ calculation. In this approach, the nanomorphology index is determined based on the distance between the representative point of the branched alkane or cycloalkane and the reference $n$-alkane straight line, $\Delta \mathrm{G}_{\mathrm{a}} \mathrm{M}$ (Equation (6)) [21].

$$
\mathrm{IM} \chi_{\mathrm{T}}=\mathrm{e}^{\frac{-\Delta \mathrm{G}_{\mathrm{M}}^{\mathrm{M}}}{\mathrm{RT}}}
$$

When the solid surface roughness is low, the $\mathrm{IM} \chi_{\mathrm{T}}$ value tends to 1 , and when the surface roughness increases, the $\mathrm{IM} \chi_{\mathrm{T}}$ value decreases due to the limited access of the branched alkanes or cycloalkanes to the rough parts of the solid surface compared to the linear $n$-alkanes.

\section{Results and Discussion}

\subsection{Sample Characterization by X-ray Diffraction and Thermogravimetry}

The sepiolite samples (Sep. 1 and Sep. 2) and the palygorskite sample (Pal) were previously analyzed by X-ray diffraction to evaluate the mineral phases present. Sep. 1 and Sep. 2 showed the presence of only sepiolite; Pal was mainly composed of palygorskite and quartz, with minor amounts of $\mathrm{Ca}-\mathrm{Mg}$-smectite, apatite-rich phase, calcite, dolomite, opal-CT, and sepiolite (Figure 1).

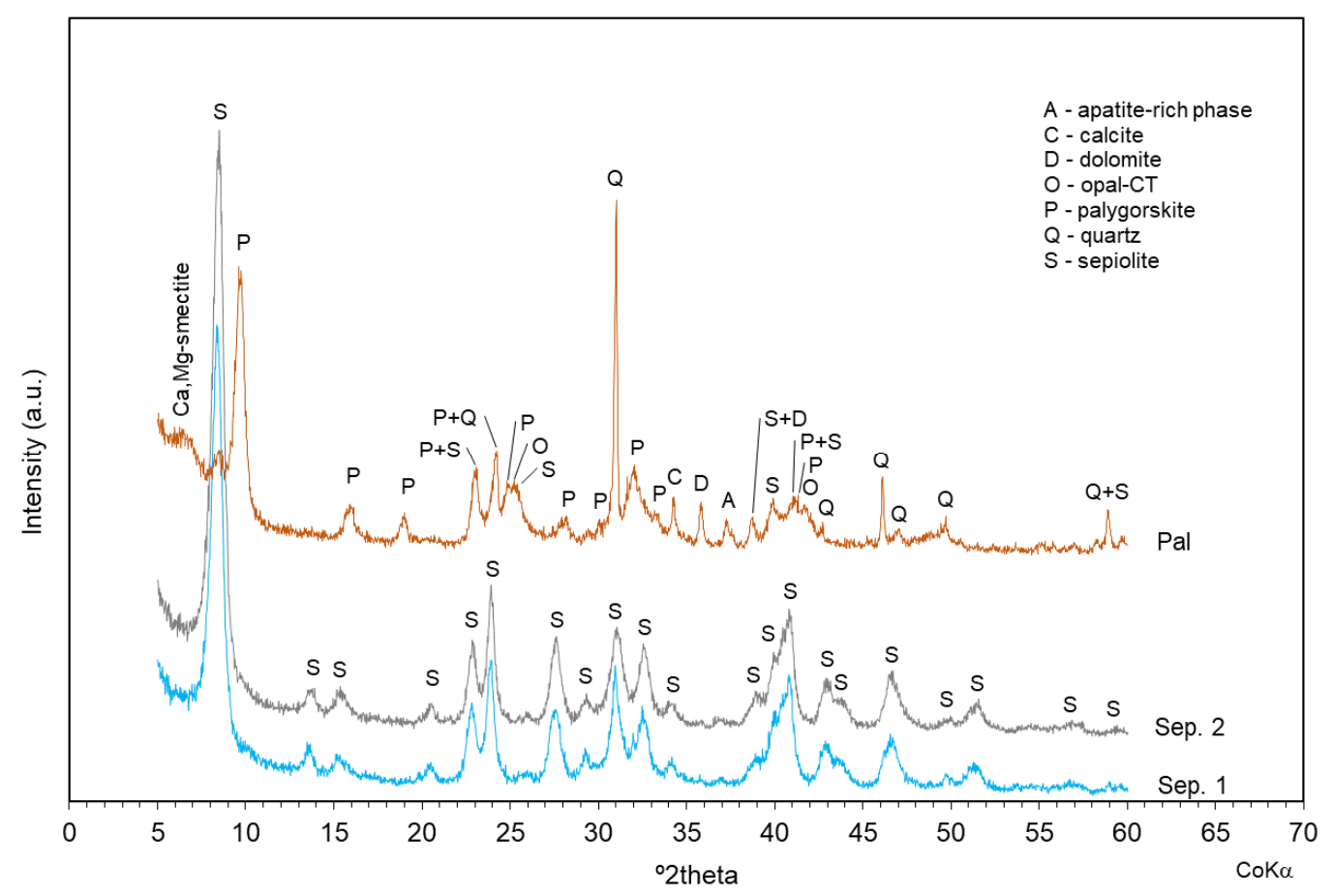

Figure 1. Mineralogical characterization of sepiolite and palygorskite samples by X-ray diffraction.

In order to choose the most suitable conditioning temperature for the IGC analyses, a thermal analysis was previously carried out. Based on the thermogravimetry (Figure S1) 
and the corresponding derivative (Figure 2), it was observed that the two sepiolite samples had quite similar thermal behavior, while the palygorskite sample exhibited a differing thermal behavior. In order to allow the removal of zeolitic water, temperatures of around $140{ }^{\circ} \mathrm{C}$, i.e., after the first step of mass loss in the thermogravimetry, were firstly selected. However, when performing the conditioning followed by the analysis at this temperature, retention times of the probes, even of the short-chain $n$-alkanes, were too long to be measured, which did not allow the analysis. Then, temperatures after the second step of mass loss were selected, which according to the values of mass loss registered would correspond to the release of all zeolitic water and more 1-2 water molecules coordinated in the structure. The conditioning temperature (Temp. cond.) was therefore $240{ }^{\circ} \mathrm{C}$ for palygorskite and $290^{\circ} \mathrm{C}$ for sepiolites (Figure 2), which afforded the removal of all zeolitic water, although some fraction of coordinated water was removed as well. To compare the studied materials under identical operational conditions, the IGC analyses were performed at a measurement temperature of $240^{\circ} \mathrm{C}$.

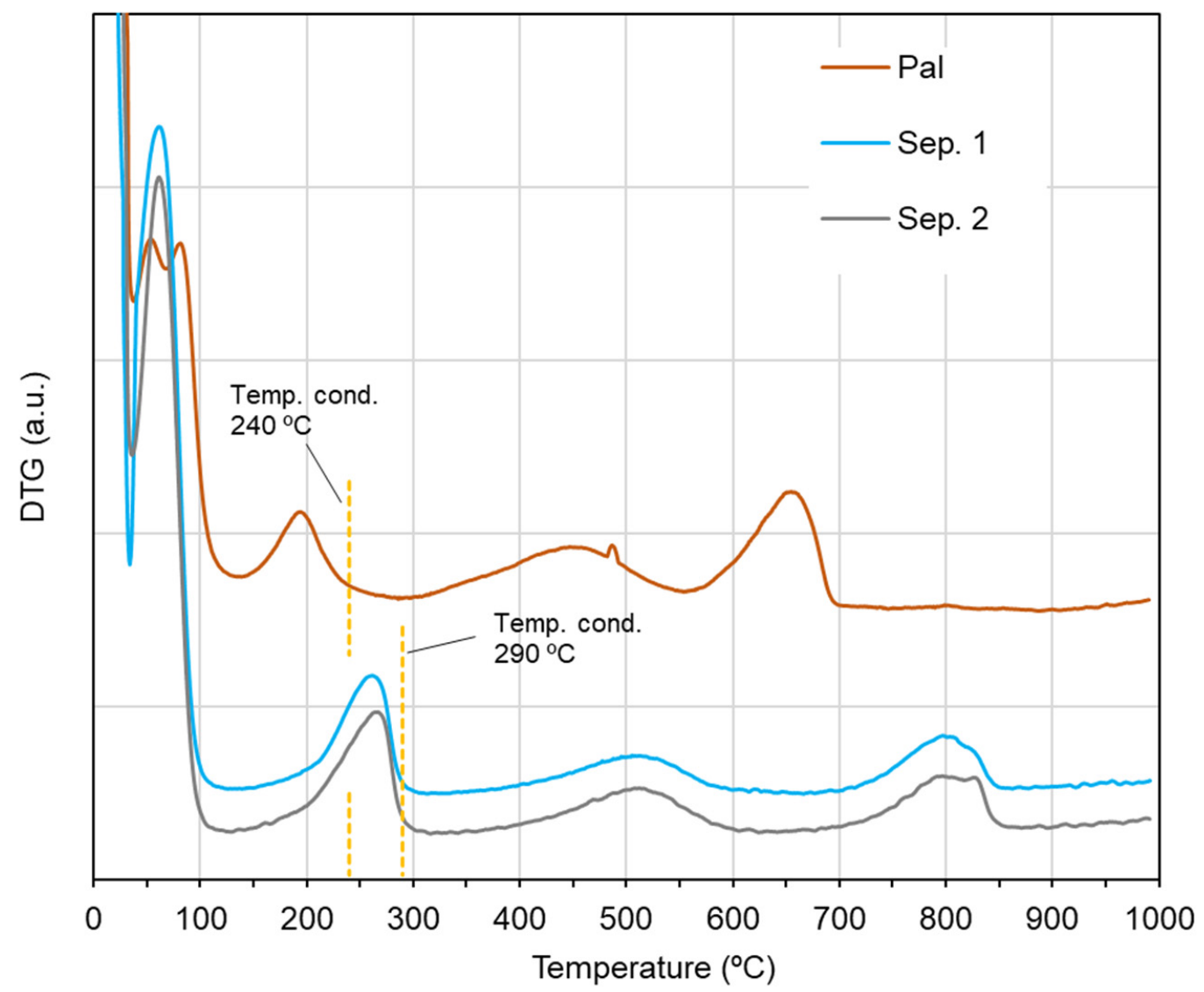

Figure 2. Derivative of the thermogravimetry (DTG) for the sepiolite and palygorskite samples.

The specific surface area was also determined, and the values of 288,337 , and $129 \mathrm{~m}^{2} \mathrm{~g}^{-1}$ were found for Sep. 1, Sep. 2, and Pal, respectively. A detailed and exhaustive characterization of the studied samples is reported by Alves et al. (2020) and Ferraz et al. (2021) [14,15].

\subsection{Dispersive Component of the Surface Free Energy}

The dispersive component of the surface free energy $\left(\gamma_{\mathrm{s}}{ }^{\mathrm{d}}\right)$ was determined from the retention times of $C_{5}$ to $C_{8} n$-alkanes by the Schultz-Lavielle and Dorris-Gray methods. The obtained results are presented in Table 1. 
Table 1. Dispersive component of the surface free energy of sepiolite and palygorskite samples at $240{ }^{\circ} \mathrm{C}$.

\begin{tabular}{|c|c|c|}
\hline Sample & $\begin{array}{c}\gamma_{\mathrm{s}}^{\mathrm{d}}(\text { Schultz-Lavielle }) \\
\left(\mathrm{mJ} \mathrm{m}^{-2}\right)\end{array}$ & $\begin{array}{c}\gamma_{\mathrm{s}}^{\mathrm{d}}(\text { Dorris-Gray) } \\
\left(\mathrm{mJ} \mathrm{m}^{-2}\right)\end{array}$ \\
\hline Sep. 1 & $135 \pm 11$ & $205 \pm 16$ \\
\hline Sep. 2 & $151 \pm 4$ & $229 \pm 6$ \\
\hline Pal & $110 \pm 5$ & $166 \pm 8$ \\
\hline
\end{tabular}

Firstly, it can be seen in Table 1 that the $\gamma_{\mathrm{s}}{ }^{\mathrm{d}}$ values obtained by the Dorris-Gray method are much higher than those obtained by the Schultz-Lavielle method (by approximately $50 \%$ ). The comparison of $\gamma_{\mathrm{s}}{ }^{\mathrm{d}}$ values obtained by these two methods was also conducted previously by Gamelas et al. (2014), where the authors studied the surface properties of calcined kaolinitic clays by IGC. For measurements performed at $110^{\circ} \mathrm{C}$, the DorrisGray method provided values ca. 20\% larger than those obtained by the Schultz-Lavielle method [22]. Additionally, Gamelas and Martins (2015), when analyzing carbonated and noncarbonated hydroxyapatites, noted that when the analysis temperature was $37{ }^{\circ} \mathrm{C}$, the difference of the results between the two methods was very small (ca. $1 \mathrm{~mJ} \mathrm{~m}^{-2}$ ), but when the analysis temperature increased to $100{ }^{\circ} \mathrm{C}$, this difference increased to about $5 \mathrm{~mJ} \mathrm{~m}{ }^{-2}$ [23]. It can be concluded that the difference in the $\gamma_{\mathrm{s}}{ }^{\mathrm{d}}$ values obtained by the two mentioned methods is inherent to the calculation approaches employed and increases when the measurement temperature increases. In the present study, since the measurement temperature was very high $\left(240{ }^{\circ} \mathrm{C}\right)$, the difference between the $\gamma_{\mathrm{s}}{ }^{\mathrm{d}}$ values obtained by these two methods was remarkably high. Additionally, it was found that the $\gamma_{\mathrm{s}}{ }^{\mathrm{d}}$ values of the three studied samples increased in the following order: Pal < Sep. $1<$ Sep. 2. Moreover, during the injections of the $n$-alkanes, the retention times of these probes were significantly longer for sepiolite samples (for similar carrier gas flow rate), which was translated into higher dispersive component values for the sepiolites. These results, for similar measurement temperature, can be attributed to the larger dimensions of the structural tunnels and channels of sepiolite, which enable higher retention of the $n$-alkane probes. When comparing the two sepiolites, the more extensive deagglomeration of the fiber bundles in Sep. 2, with a higher specific surface area, may be the reason for a somewhat higher $\gamma_{s}{ }^{d}$ value compared to Sep. 1. The $\gamma_{s}{ }^{d}$ values obtained in this study for the sepiolite samples (205 and $229 \mathrm{~mJ} \mathrm{~m}^{-2}$ for Sep. 1 and Sep. 2, respectively) appear to be higher than those found in the literature [10-12]. In particular, the present results were higher when compared specifically with the results of Lazarević et al. (2009), who studied sepiolite by IGC, between 210 and $240^{\circ} \mathrm{C}$, and obtained a dispersive component value (by Dorris-Gray method) of $130 \mathrm{~mJ} \mathrm{~m}^{-2}$ at $240{ }^{\circ} \mathrm{C}$ [12]. Despite the fact that they used the same temperature as ours, differences in geology and pretreatment of the sepiolites can be the origin of such different results in the dispersive component. Boudriche et al. (2010) also analyzed a palygorskite sample by IGC and obtained a dispersive component value very similar to ours $\left(164 \mathrm{~mJ} \mathrm{~m}^{-2}\right.$ at $\left.130^{\circ} \mathrm{C}\right)$ [13]. However, since the operating temperature used by Boudriche and coworkers was much lower than ours, and due to differences in the pretreatment and in the mineral composition of the palygorskite samples, an accurate comparison with this result cannot be made.

\subsection{Specific Interaction Parameters}

As mentioned in Section 2.2, typical Lewis basic and amphoteric probes such as tetrahydrofuran, diethyl ether, ethyl acetate, or acetone were retained too strongly in the studied clay materials and could not be eluted through the chromatographic columns. Thus, in order to be able to measure the Lewis acidic character of the mineral surface, weak Lewis bases (1-alkenes) were injected into the IGC column. In addition to these probes, cycloalkanes (cyclohexane and cyclooctane) and a branched alkane (2,2,4-trimethylpentane) were also injected. The specific interactions of adsorption $\left(-\Delta \mathrm{G}_{\mathrm{a}}{ }^{\mathrm{s}}\right)$ of the injected probes on the surface of the studied materials were determined by the difference between the 
$R T \ln \left(V_{n}\right)$ values of 1-alkenes, cycloalkanes, or branched alkane and the $R T \ln \left(V_{n}\right)$ values of the corresponding $n$-alkanes with the same number of carbon atoms. It should be noted that, actually, for the cycloalkanes or the branched alkane, the intermolecular forces with the solid material are of similar nature to those involving the $n$-alkanes. Therefore, in these cases, the "specific" interaction does not correspond to an additional interaction to the dispersive Lifshitz-van der Waals forces but rather is a negative contribution, as demonstrated below. The results are shown in Figure 3.

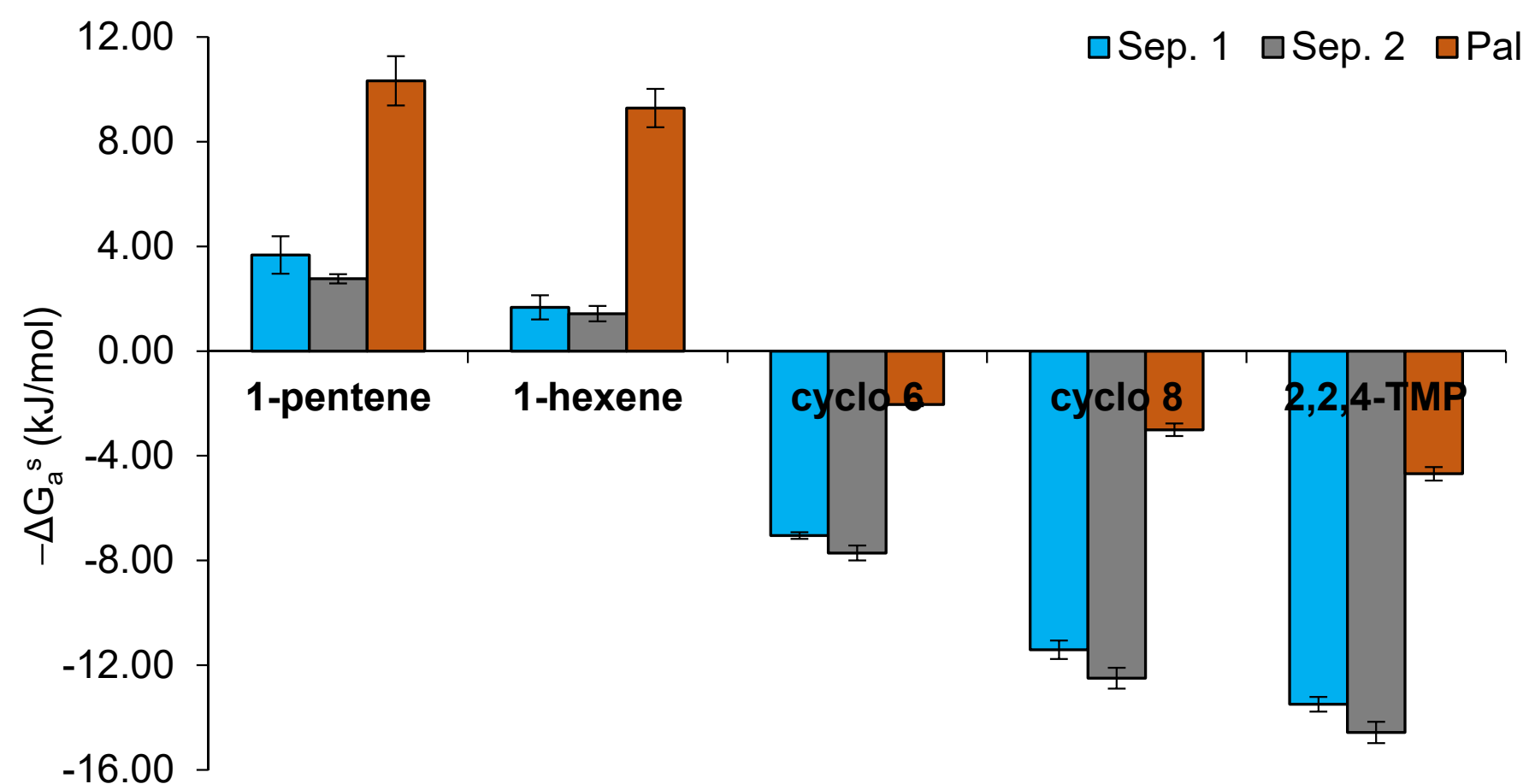

Figure 3. Specific interaction parameter $\left(-\Delta \mathrm{G}_{\mathrm{a}} \mathrm{s}, \mathrm{kJ} \mathrm{mol}^{-1}\right)$ for the adsorption of 1-alkenes (1-pentene and 1-hexene), cycloalkanes (cyclohexane (cyclo 6) and cyclooctane (cyclo 8)), and a branched alkane (2,2,4-trimethylpentane (2,2,4-TMP)) on the surface of sepiolite and palygorskite samples at $240^{\circ} \mathrm{C}$.

The specific interactions with the $\pi$ electron donor bases (1-alkenes) were significantly higher for palygorskite (near $10 \mathrm{~kJ} \mathrm{~mol}^{-1}$ ) than for sepiolite samples (did not exceed $4 \mathrm{~kJ} \mathrm{~mol}^{-1}$ ), suggesting that the surface of the palygorskite particles showed the most pronounced Lewis acidic character of the three studied samples (Figure 3). Additionally, a slight decrease in the $-\Delta \mathrm{G}_{\mathrm{a}}{ }^{\mathrm{s}}$ values of 1-alkenes was observed with the increase in the carbon atom number. This trend has already been reported for other mineral materials [24]. To our knowledge, specific interactions of weak Lewis bases with sepiolite and palygorskite surfaces were never studied. Comparing to other minerals, the present values determined for palygorskite were higher than those reported for calcined kaolinitic clays and hydroxyapatites $\left(3-4 \mathrm{~kJ} \mathrm{~mol}^{-1}\right)[22,23]$. As mentioned above, the Lewis acid-base probes more typically used in IGC could not be measured due to their strong interactions with the present samples, which means that the sepiolite and palygorskite surfaces under study have a strong amphoteric character. Other authors have already evaluated the acid-base properties of these two types of clay minerals by IGC, by injecting the more common Lewis acid-base probes and measuring their interactions, whenever possible. In the case of sepiolite, both acidic [10,11] and basic [12] characters have already been reported. For palygorskite, Boudriche et al. (2010) mentioned a strongly acidic character because of the fact that they could not elute basic probes (due to very strong interactions with the palygorskite surface) [13].

The cycloalkane probes (cyclo 6 and cyclo 8 ) showed significantly lower retention times than their corresponding $n$-alkanes ( $n$-hexane and $n$-octane), and, accordingly, the 
specific interactions of these probes were always negative for all samples (Figures 3 and 4). This fact can be explained by steric hindrance effects of cycloalkanes which limit their access to the structural channels of sepiolite and palygorskite when compared with $n$-alkanes [25]. The additional two carbon atoms in the cyclooctane ring, compared to cyclohexane, confer a higher volume to this probe, and, consequently, its relative access to the channels is more limited (retention time of cyclooctane vs. $n$-octane is speeded up compared to retention time of cyclohexane vs. $n$-hexane), providing even more negative specific interactions than cyclohexane. The specific interaction values of both cycloalkanes with the studied samples increased in the following order: Sep. $2<$ Sep. $1<<$ Pal. This trend is in agreement with the smaller dimensions of the structural channels of palygorskite and with the different pretreatments applied to the sepiolite samples. Finally, the 2,2,4-TMP kept the same behavior as that observed for the cycloalkanes (Figure 3). However, the $-\Delta \mathrm{G}_{\mathrm{a}}{ }^{\mathrm{s}}$ values, which were obtained by difference between the $\mathrm{RT} \ln \left(\mathrm{V}_{\mathrm{n}}\right)$ values of 2,2,4-TMP and $n$-octane, were even more negative due to the branched structure of the chain of this probe.

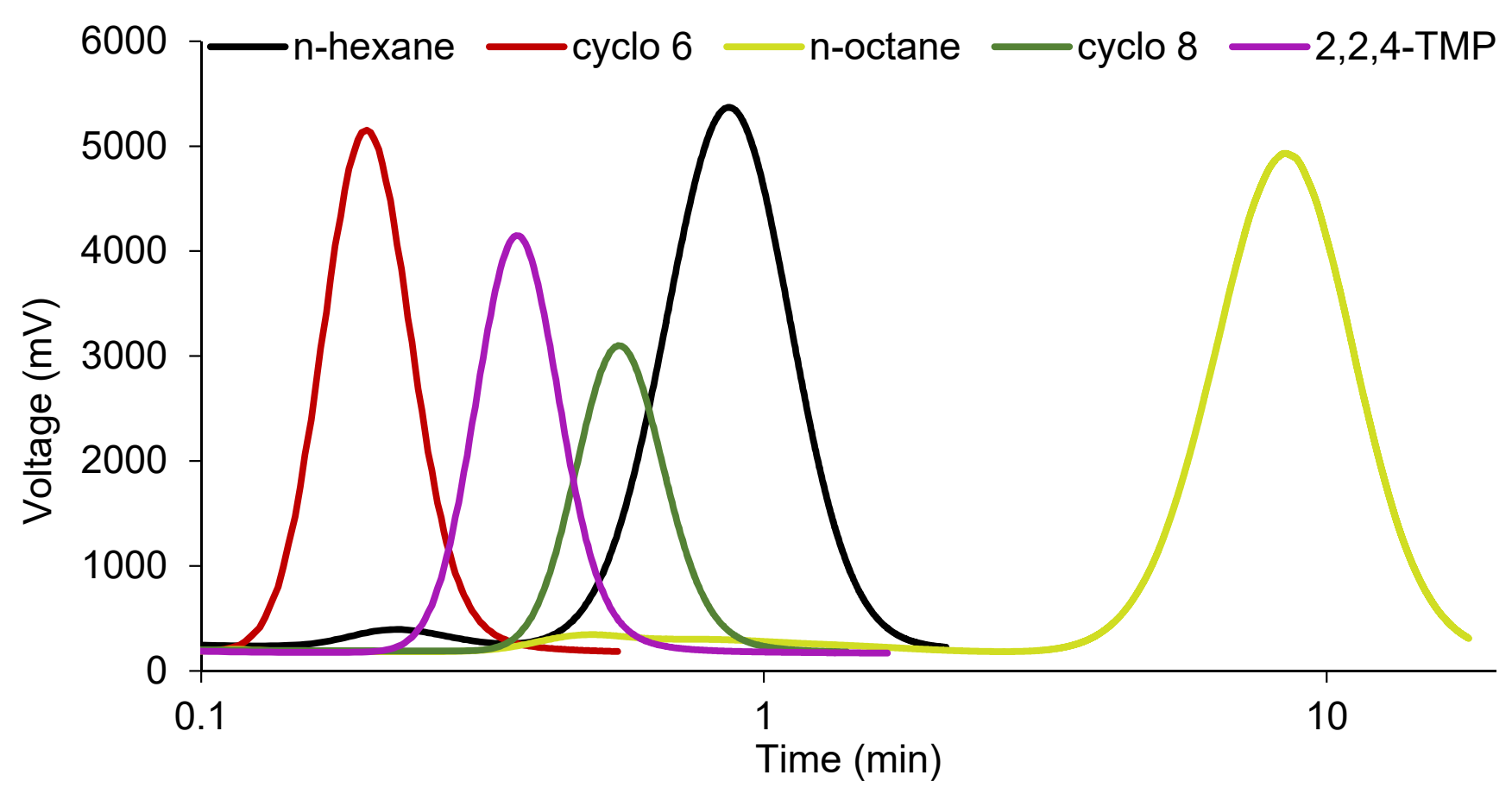

Figure 4. Examples of chromatograms for the elution of $n$-hexane, cyclohexane (cyclo 6), $n$-octane, cyclooctane (cyclo 8), and 2,2,4-trimethylpentane (2,2,4-TMP) in sepiolite 2 at $240{ }^{\circ} \mathrm{C}$ (flow rate of $63 \mathrm{~mL} \mathrm{~min}^{-1}$ ). Due to large differences between probes in the values of retention times, these are shown on a logarithmic scale for a better visualization.

\subsection{Nanomorphology Index $\left(\operatorname{IM} \chi_{T}\right)$}

In order to assess the surface nanoroughness of the studied clay minerals, the nanomorphology indices $\left(\mathrm{IM} \chi_{\mathrm{T}}\right)$ were determined based on the measurement of retention times of cycloalkanes (cyclo 6 and cyclo 8 ) and of a branched alkane (2,2,4-TMP), according to two different approaches. The results are shown in Table 2.

Table 2. Nanomorphology indices $\left(\mathrm{IM} \chi_{\mathrm{T}}\right)$, as calculated by two methods, of sepiolite and palygorskite samples at $240{ }^{\circ} \mathrm{C}$.

\begin{tabular}{ccccccc}
\hline Probes & \multicolumn{2}{c}{ IM $\boldsymbol{\chi}_{\mathbf{T}} \mathbf{( \% , ~ B r e n d l e ́ ~ a n d ~ P a p i r e r , ~ 1 9 9 7 ) ~}$} & \multicolumn{3}{c}{ IM $\boldsymbol{\chi}_{\mathbf{T}}$ (Balard et al. 2000) } \\
\hline & Sep. 1 & Sep. 2 & Pal & Sep. 1 & Sep. 2 & Pal \\
Cyclo 6 & $-24.1 \pm 1.7$ & $-25.7 \pm 0.4$ & $-9.6 \pm 0.4$ & $0.16 \pm 0.010$ & $0.15 \pm 0.003$ & $0.53 \pm 0.010$ \\
Cyclo 8 & $-30.0 \pm 0.6$ & $-32.9 \pm 0.5$ & $-11.7 \pm 0.5$ & $0.05 \pm 0.005$ & $0.04 \pm 0.003$ & $0.35 \pm 0.013$ \\
2,2,4-TMP & $-26.6 \pm 1.2$ & $-30.0 \pm 0.5$ & $-5.6 \pm 0.7$ & $0.09 \pm 0.002$ & $0.07 \pm 0.005$ & $0.64 \pm 0.030$ \\
\hline
\end{tabular}


The two different approaches used for the determination of $\mathrm{IM} \chi_{\mathrm{T}}$ led to the same trends: more negative values of $\mathrm{IM} \chi_{\mathrm{T}}$ calculated by the Brendlé and Papirer approach correspond to values closer to zero when calculated by the Balard approach, that is, a material with a rougher surface. Based on the results presented in Table 2, it can then be observed that Sep. 2 had the most negative values (in the Brendle and Papirer approach) and the ones closest to zero (in the Balard approach) for each probe assessed, meaning that Sep. 2 had the roughest surface of all samples. However, the differences from the Sep. 1 sample were not high. On the contrary, Pal showed, by a large difference, the surface with the lowest roughness. From this trend, it was concluded that a higher surface nanoroughness correlated to a higher dispersive component of the surface free energy. This observation was also reported previously by Saada et al. (1995) in the evaluation of the surface properties of illites and kaolinites by IGC [26] and for calcined kaolinitic clays [22]. Rougher surfaces with increasing structural surface defects at the molecular/nanometric scale provide a better insertion of the $n$-alkanes into the material structure. Boudriche et al. (2010) also determined the nanomorphology indices (Balard approach) for a palygorskite sample with cyclooctane and 2,3,4-TMP and obtained IM $\chi_{\mathrm{T}}$ values of 0.22 and 0.69 , respectively [13]. Comparing the IM $\chi_{\mathrm{T}}$ values of cyclooctane, it can be said that our palygorskite sample showed a flatter surface.

\section{Commercial Applications of the Mineral Samples and Relevance of IGC Results}

At present time, regarding the studied samples, sepiolite 1 is used as a sorbent and carrier for chemicals; to control the flow of some fluid systems, such as bitumen sheets, particularly at higher temperatures; and as semireinforcing mineral filler for rubber and polar polymers. Sepiolite 2 is used to provide a thixotropic and pseudoplastic behavior to water-based paints and coatings; as a suspending agent to avoid settling of pigments and fillers; and as a reinforcing filler for polar polymers. When surface-modified, sepiolite 2 can be incorporated in different polymer compounds to improve mechanical properties and fire resistance. Palygorskite is also used as a rheological additive, particularly for building products, such as mortars, wet and dry joint compounds, adhesives, and sealants. It provides a shear-thinning rheological behavior; improves pumpability, workability, and spreading of the compositions; and improves sag resistance when applied in vertical walls.

The present results obtained by IGC demonstrate the high potential of the studied mineral samples for the compatibilization with polymeric matrices with high polarity (e.g., polysaccharide and polyacrylate matrices) and adsorption of a wide variety of substances present in liquid and gaseous effluent streams (e.g., formaldehyde, chlorine- and sulfurcontaining compounds).

\section{Conclusions}

In this work, two sepiolite samples, with different pretreatments, and one palygorskite sample were extensively characterized by inverse gas chromatography (IGC) and compared. Based on a previous analysis by X-ray diffraction, the two sepiolite samples were found to have a similar mineralogical composition, as expected, with the presence of only sepiolite. On the other hand, the palygorskite sample was more heterogeneous, composed not only of palygorskite but also of various contaminants such as quartz.

The results obtained by IGC at $240^{\circ} \mathrm{C}$, after previous preconditioning of all samples for the removal of all zeolitic water, revealed that sepiolite samples had higher $\gamma_{\mathrm{s}}{ }^{\mathrm{d}}$ values than palygorskite, which was related to the larger dimensions of the tunnels and channels present in the structure of the sepiolite vs. palygorskite. It was also observed that the $\gamma_{\mathrm{s}}{ }^{\mathrm{d}}$ values of sepiolites were slightly affected by the different applied pretreatments. The nonelution of typical Lewis basic, amphoteric, or acidic probes revealed very strong interactions of those probes with the studied minerals and allowed us to conclude that the analyzed sepiolite and palygorskite surfaces have a very strong amphoteric character. On the other hand, the injections of cyclohexane, cyclooctane, and 2,2,4-trimethylpentane showed that steric hindrance of these cyclic and branched alkanes makes it difficult for 
them to reach the structural channels of the minerals. The nanomorphology indices $\left(\operatorname{IM} \chi_{T}\right)$, calculated based on the retention times of the latter set of probes, showed that the sepiolites clearly exhibited a higher nanoroughness compared to the palygorskite sample, which behaved more like a flatter surface.

It should be highlighted that the very high values of surface energy and the developed amphoteric character observed for the sepiolite and palygorskite samples under study, together with their high specific surface area, even compared with other samples of these minerals previously reported, may favor adsorption processes with volatile organic compounds or matrix-filler interactions regarding the production of composite structures with matrices able to establish Lewis acid-base interactions.

Supplementary Materials: The following are available online at https: / www.mdpi.com/article / 10.3390/nano11061579/s1. Figure S1, Thermogravimetry (mass loss) for the sepiolite and palygorskite samples.

Author Contributions: Conceptualization, J.A.F.G.; methodology, R.A. and J.A.F.G.; investigation, R.A., E.F. and J.A.F.G.; writing-original draft preparation, R.A. and J.A.F.G.; writing-review and editing, R.A., E.F., J.S. and J.A.F.G.; supervision, J.A.F.G.; project administration, J.A.F.G.; funding acquisition, J.A.F.G. All authors have read and agreed to the published version of the manuscript.

Funding: The present research was supported by the R\&D project titled "FILCNF-New generation of composite films of cellulose nanofibrils with mineral particles as high strength materials with gas barrier properties" (PTDC/QUI-OUT/31884/2017, CENTRO 01-0145-FEDER-031884), funded by the Fundação para a Ciência e Tecnologia (FCT) and FEDER. Strategic Research Centre Project (UIDB/00102/2020) and Techn\&Art Project (UID/05488/2018) funded by the FCT are also acknowledged.

Data Availability Statement: The data presented in this study are available on request from the corresponding authors. The data are not publicly available due to lack of adequate repository.

Conflicts of Interest: The authors declare no conflict of interest.

\section{References}

1. Cecilia, J.A.; Vilarrasa-García, E.; Cavalcante, C.L.; Azevedo, D.C.S.; Franco, F.; Rodríguez-Castellón, E. Evaluation of two fibrous clay minerals (sepiolite and palygorskite) for $\mathrm{CO}_{2}$ capture. J. Environ. Chem. Eng. 2018, 6, 4573-4587. [CrossRef]

2. Singer, A. Palygorskite and Sepiolite Group Minerals. In Minerals in Soil Environments, 2nd ed.; Dixon, J.B., Weed, S.B., Eds.; Soil Science Society of America: Madison, WI, USA, 1989; Volume 1, pp. 829-872.

3. Suaréz, M.; García-Romero, E. Chapter 2-Advances in the Crystal Chemistry of Sepiolite and Palygorskite. In Developments in Clay Science; Galán, E., Singer, A., Eds.; Elsevier: Amsterdam, The Netherlands, 2011; Volume 3, pp. 33-65. [CrossRef]

4. Galán, E. Properties and applications of palygorskite-sepiolite clays. Clay Miner. 1996, 31, 443-453. [CrossRef]

5. Ruiz-Hitzky, E.; Darder, M.; Fernandes, F.M.; Wicklein, B.; Alcântara, A.C.S.; Aranda, P. Fibrous clays based bionanocomposites. Prog. Polym. Sci. 2013, 38, 1392-1414. [CrossRef]

6. Santos, J.M.R.C.A.; Guthrie, J.T. Analysis of interactions in multicomponent polymeric systems: The key-role of inverse gas chromatography. Mater. Sci. Eng. R Rep. 2005, 50, 79-107. [CrossRef]

7. Gamelas, J.A.F. The surface properties of cellulose and lignocellulosic materials assessed by inverse gas chromatography: A review. Cellulose 2013, 20, 2675-2693. [CrossRef]

8. Mohammadi-Jam, S.; Waters, K.E. Inverse gas chromatography applications: A review. Adv. Colloid Interface Sci. 2014, 212, 21-44. [CrossRef]

9. Gamelas, J.A.F.; Salvador, A.; Hidalgo, J.; Ferreira, P.J.; Tejado, A. Unique combination of surface energy and lewis acid-base characteristics of superhydrophobic cellulose fibers. Langmuir 2017, 33, 927-935. [CrossRef]

10. Morales, E.; Dabrio, M.V.; Herrero, C.R.; Acosta, J.L. Acid/base characterization of sepiolite by inverse gas chromatography. Chromatographia 1991, 31, 357-361. [CrossRef]

11. Așkin, A.; Yazici, D.T. Surface characterization of sepiolite by inverse gas chromatography. Chromatographia 2005, 61, 625-631. [CrossRef]

12. Lazarević, S.; Radovanović, Ž.; Veljović, D.; Onjia, A.; Janaćković, D.; Petrović, R. Characterization of sepiolite by inverse gas chromatography at infinite and finite surface coverage. Appl. Clay Sci. 2009, 43, 41-48. [CrossRef]

13. Boudriche, L.; Hamdi, B.; Kessaïssia, Z.; Calvet, R.; Chamayou, A.; Dodds, J.A.; Balard, H. An assessment of the surface properties of milled attapulgite using inverse gas chromatography. Clays Clay Miner. 2010, 58, 143-153. [CrossRef]

14. Alves, L.; Ferraz, E.; Santarén, J.; Rasteiro, M.G.; Gamelas, J.A.F. Improving colloidal stability of sepiolite suspensions: Effect of the mechanical disperser and chemical dispersant. Minerals 2020, 10, 779. [CrossRef] 
15. Ferraz, E.; Alves, L.; Sanguino, P.; Santarén, J.; Rasteiro, M.G.; Gamelas, J.A.F. Stabilization of palygorskite aqueous suspensions using bio-based and synthetic polyelectrolytes. Polymers 2021, 13, 129. [CrossRef] [PubMed]

16. Kamdem, D.P.; Riedl, B. Inverse gas chromatography of lignocellulosic fibers coated with a thermosetting polymer: Use of peak maximum and Conder and Young methods. J. Colloid Interface Sci. 1992, 150, 507-516. [CrossRef]

17. Mukhopadhyay, P.; Schreiber, H.P. Aspects of acid-base interactions and use of inverse gas chromatography. Colloids Surf. A Physicochem. Eng. Asp. 1995, 100, 47-71. [CrossRef]

18. Schultz, J.; Lavielle, L.; Martin, C. The role of the interface in carbon fibre-epoxy composites. J. Adhes. 1987, 23, 45-60. [CrossRef]

19. Dorris, G.M.; Gray, D.G. Adsorption of $n$-alkanes at zero surface coverage on cellulose paper and wood fibres. J. Colloid Interface Sci. 1980, 77, 353-362. [CrossRef]

20. Brendlé, E.; Papirer, E. A new topological index for molecular probes used in inverse gas chromatography for the surface nanorugosity evaluation. J. Colloid Interface Sci. 1997, 194, 207-216. [CrossRef]

21. Balard, H.; Brendlé, E.; Papirer, E. Determination of Acid-Base properties of Solids Surfaces using Inverse Gas Chromatography: Advantages and Limitations. In Acid- Base Interactions: Relevance to Adhesion Science and Technology; Mittal, K.L., Ed.; VSP: Utrecht, The Netherlands, 2000; Volume 2, pp. 299-316.

22. Gamelas, J.A.F.; Ferraz, E.; Rocha, F. An insight into the surface properties of calcined kaolinitic clays: The grinding effect. Colloids Surf. A Physicochem. Eng. Asp. 2014, 455, 49-57. [CrossRef]

23. Gamelas, J.A.F.; Martins, A.G. Surface properties of carbonated and non-carbonated hydroxyapatites obtained after bone calcination at different temperatures. Colloids Surf. A Physicochem. Eng. Asp. 2015, 478, 62-70. [CrossRef]

24. Perruchot, C.; Chehimi, M.M.; Vaulay, M.J.; Benzarti, K. Characterisation of the surface thermodynamic properties of cement components by inverse gas chromatography at infinite dilution. Cem. Concr. Res. 2006, 36, 305-319. [CrossRef]

25. Ansari, D.M.; Price, G.J. Chromatographic estimation of filler surface energies and correlation with photodegradation of kaolin filled polyethylene. Polymer 2004, 45, 1823-1831. [CrossRef]

26. Saada, A.; Papirer, E.; Balard, H.; Siffert, B. Determination of the surface properties of illites and kaolinites by inverse gas chromatography. J. Colloid Interface Sci. 1995, 175, 212-218. [CrossRef] 